\title{
Cálculo de Estimativas para Valores Futuros do Preço Spot da Energia Elétrica no Brasil Utilizando Análise de Sensibilidade
}

\author{
Calculation of Estimates for Future Values of Energy Spot Price in Brazil Using \\ Sensitivity Analysis
}

Mário Cavalcanti Gameiro de Moura Filho ${ }^{1}$

\section{Eduardo de Aguiar Sodré1}

${ }^{1}$ Escola Politécnica de Pernambuco, Universidade de Pernambuco, Recife, Brasil.

E-mail do autor principal: Mário C. G. de Moura Filho mario_gameiro77@hotmail.com

\section{RESUMO}

Após a reformulação do setor elétrico Brasileiro e a criação do Ambiente de Contratação Livre (ACL), no início dos anos 2000, a figura do agente comercializador se fez muito mais presente na estrutura do mercado de energia. Conforme as regras atuais de comercialização de energia elétrica, e para o devido atendimento dos consumidores e melhores resultados financeiros dos agentes envolvidos em negociações no $A C L$, a avaliação futura dos preços da energia no Mercado Spot (também conhecido como Mercado de Curto Prazo) é de fundamental importância. No Brasil, este preço é denominado Preço de Liquidação das Diferenças (PLD) e são utilizados modelos matemáticos de otimização para sua determinação: o NEWAVE para médio prazo; e o modelo DECOMP para o curto prazo. Neste trabalho é realizada uma análise de sensibilidade para projeção futura de valores do PLD. Também se exemplificou a importância do monitoramento e do impacto dos seguintes fatores que influenciam a formação do preço: afluências; armazenamento dos reservatórios; e carga de energia elétrica. Para as análises quantitativas foram utilizados o deck do DECOMP disponibilizados pela Câmara de Comercialização de Energia.

PALAVRAS-ChAVE: DECOMP, NEWAVE, PLD, Análise de Sensibilidade;

\section{ABSTRACT}

After the reformulation of the Brazilian Electricity Sector and the creation of the Free Contracting Environment (FCE), in the early 2000s, the figure of the commercialization agent became much more present in the structure of the energy market. In accordance with the current rules for the commercialization of electricity, and for the consumer's attendance and better financial results of the agents involved in negotiations in the $A C L$, the future assessment of energy prices in the Spot Market (also known as Short-term Market) is of extremely importance. In Brazil, this price is denominated Differences Liquidation Price (DLP) and mathematical models of optimization are used for its determination: NEWAVE for medium term; and the DECOMP model for the short term. In this work a sensitivity analysis is performed for future projection of DLP values. We also exemplify the importance of monitoring and the impact of the following factors that influence price formation: inflows; storage of reservoirs; and electrical power load. For the quantitative analyzes, the deck of the DECOMP made available by the Chamber of Electrical Energy Commercialization was used.

KEY-WORDS:DECOMP, NEWAVE, PLD, Sensitivity Analysis. 


\section{INTRODUÇÃO}

A crise energética ocorrida nos anos 90 forçou uma reformulação do setor elétrico brasileiro visando à entrada de capital privado e a criação de novos agentes, onde o governo assumisse o papel de agente orientador e regulador. Em 1996, através do Projeto de Reestruturação do Setor Elétrico Brasileiro (RE-SEB), deu-se início à criação de um novo modelo para o setor elétrico, resultando na criação da Agência Nacional de Energia Elétrica (ANEEL) como agente regulador; o Operador Nacional do Sistema Elétrico (ONS) como agente operador do sistema interligado; e um ambiente de transações, o Mercado Atacadista de Energia (MAE) através do Administrador de serviços do Mercado Atacadista de Energia (ASMAE) [1].

Após a reformulação no setor, o racionamento ocorrido no ano de 2001, fez com que a estrutura regulatório-normativa proposta incialmente fosse revista. Nesta reforma ocorrida em 2003, conhecida como o Novo Modelo, o Mercado Livre passou a se chamar Ambiente Contratação Livre $(A C L)$ e ficou direcionado a agentes geradores; agentes comercializadores; agentes importadores e exportadores; consumidores de grande porte (com carga superior a $3 \mathrm{MW}$ e atendidos em tensão superior a $69 \mathrm{KV}$ ) e consumidores especiais (consumidores que possuam carga acima de $500 \mathrm{~kW}$ atendidos em qualquer tensão) [2].

Especificamente os denominados Consumidores Especiais são aqueles consumidores que são atendidos por fontes de energia renovável, podendo ser provenientes de Pequenas Centrais Hidroelétricas ( $\mathrm{PCHs}$ ) entre $1 \mathrm{MW}$ e $30 \mathrm{MW}$, usinas solares, eólicas ou biomassa até 30MW. Para este tipo de consumidor, também é aplicado o desconto nas tarifas de uso do sistema de distribuição (TUSD) e desconto nas tarifas de uso do sistema de transmissão (TUST) [3].

A principal característica do ACL é a autonomia que os agentes possuem para negociar compra e venda de energia elétrica. Liberdade que compreende negociação de preços, prazos, montantes de energia, flexibilidade no uso, penalidades e garantias.

Dando continuidade à formulação do Novo Modelo, em março de 2004, através da lei $n^{\circ}$ 10.848 criou-se a Câmara de Comercialização de Energia Elétrica (CCEE) para substituir O MAE. Algumas das atribuições da CCEE são: 41 administração do Ambiente de Contratação Regulada (ACR) e do Ambiente de contratação Livre $(A C L)$; apuração do Preço de Liquidação das Diferenças (PLD); e contabilização e liquidação das transações realizadas no mercado de curto prazo [4].

A partir deste momento a figura do comercializador faz-se presente na estrutura do sistema elétrico, sendo um intermediador entre os geradores e consumidores como visto na Figura 1, com o objetivo de reduzir os custos de transação e permitir o atendimento dos consumidores de acordo com suas necessidades de compra de energia [5].

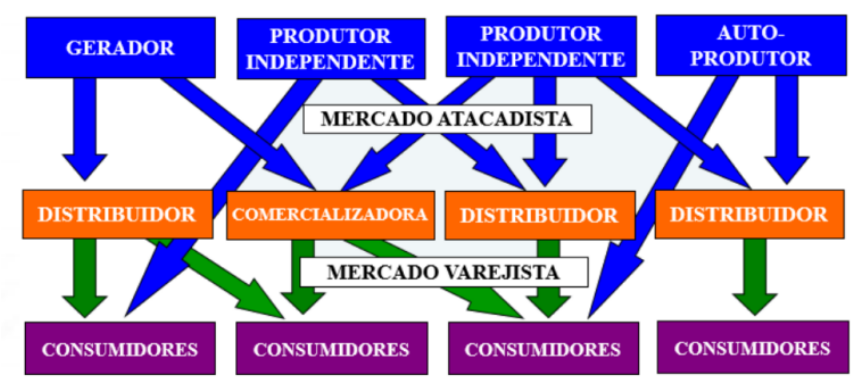

Figura 1: Agentes do mercado de Energia. Fonte: [6].

Na próxima seção serão apresentadas as definições e características básicas da formação do PLD no médio e curto prazo através da utilização dos modelos computacionais NEWAVE e DECOMP.

Tendo em vista otimizar a compra/venda de energia, o presente trabalho tem como objetivo mostrar a importância do monitoramento e projeção das variáveis (previsões de afluências, demanda de energia e armazenamento) que formam o PLD no curto prazo no modelo DECOMP para o período de abril de 2018.

\section{Formação do PLD}

O cálculo do PLD baseia-se no despacho "exante", ou seja, é apurado com base em informações previstas, anteriores à operação real do sistema, considerando-se os valores de disponibilidades declaradas de geração e o consumo previsto de cada submercado.

O processo completo de cálculo do PLD consiste na utilização dos modelos computacionais NEWAVE e DECOMP, os quais produzem como resultado o Custo Marginal de Operação (CMO) de 
cada submercado, respectivamente em base mensal e semanal [6].

Sistemas hidrotérmicos podem utilizar a energia armazenada nos reservatórios do sistema para atender a carga, substituindo a geração térmica.

Pode-se ver na Figura 2, por exemplo, que caso a decisão seja "Minimizar o custo do combustível esvaziando os reservatórios" e no futuro houver um período de seca, então inevitavelmente 0 suprimento terá que ser realizado através de usinas térmicas de custo elevado, pois a água dos reservatórios já foi utilizada no momento presente [7].

Por outro lado, caso a decisão seja por "Manter os reservatórios cheios e usar a geração térmica", e no futuro haja uma elevação nas vazões, pode ser que seja necessário o vertimento de água, o que representa um desperdício de energia e por consequência um aumento desnecessário no custo de operação [7].

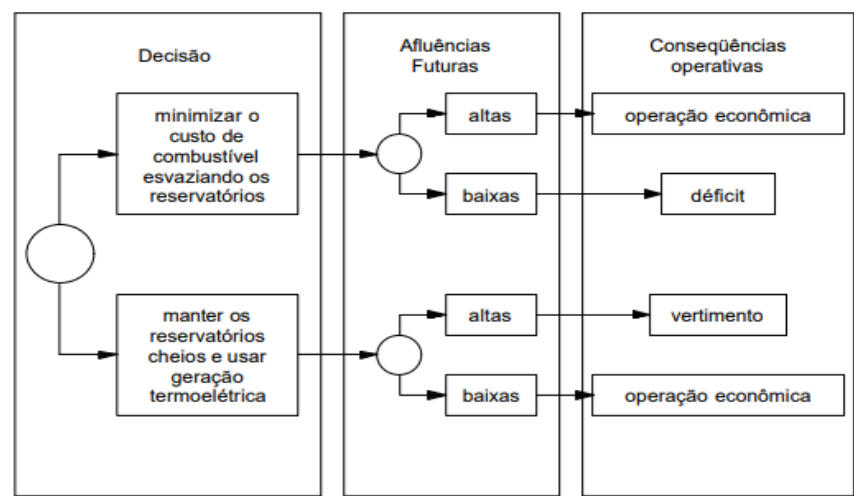

Figura 2- Processo de Decisão para sistemas hidrotérmicos. Fonte: [13].

O PLD é utilizado para liquidar a compra e a venda de energia no mercado de curto prazo. Calculado pela CCEE, é um valor determinado semanalmente para cada patamar de carga baseado no CMO. O CMO é calculado pela ONS com base nas condições hidrológicas, na demanda de energia, nos preços de combustível, no custo de déficit, na entrada de novos projetos e na disponibilidade de equipamentos de geração e transmissão, o modelo de precificação obtém o despacho (geração) ótimo para o período em estudo, definindo a geração hidráulica e a geração térmica para cada subsistema [6].

\subsection{NEWAVE}

O Modelo NEWAVE tem como objetivo realizar a alocação ótima de recursos térmicos e hídricos, visando à redução do custo total esperado do CMO [8].

O NEWAVE representa o parque gerador hidrelétrico de forma agregada, representando as usinas hidrelétricas através de um reservatório equivalente de energia. O NEWAVE é utilizado para o planejamento de médio prazo (até 5 anos), com discretização mensal [9].

O modelo NEWAVE pode ser dividido em 4 módulos, a saber:

1. Cálculo do Sistema Equivalente de Energia: Este módulo é responsável por representar as usinas em quatro subsistemas equivalentes: Sudeste/Centro-Oeste, Sul, Nordeste e Norte [10];

2. Geração de Séries Sintéticas: Este módulo é responsável por gerar as séries sintéticas de energias naturais afluentes do subsistema, baseado na série histórica de vazões. É determinando para cada subsistema a energia total afluente, que é a composta pela energia controlável e pela energia de fio d'água [10];

3. Cálculo da Política de Operação Hidrotérmica: Neste módulo é determinada a política de operação ótima do sistema equivalente através da metodologia de Programação Dinâmica Dual Estocástica (PDDE), considerando as afluências futuras [10];

4. Simulação da Operação: Neste módulo é determinando a operação do sistema no horizonte de planejamento, considerando as funções de custo futuro encontradas no módulo anterior. Risco de déficit, intercâmbios energéticos e montantes de geração hidrelétrica e térmica são algumas saídas deste módulo [11].

Um dos principais resultados desse modelo são as FCF (funções de custo futuro), exemplificada na Figura 3, que traduzem para os modelos de outras etapas (de curto prazo) o impacto da utilização da água armazenada nos reservatórios [12]. 


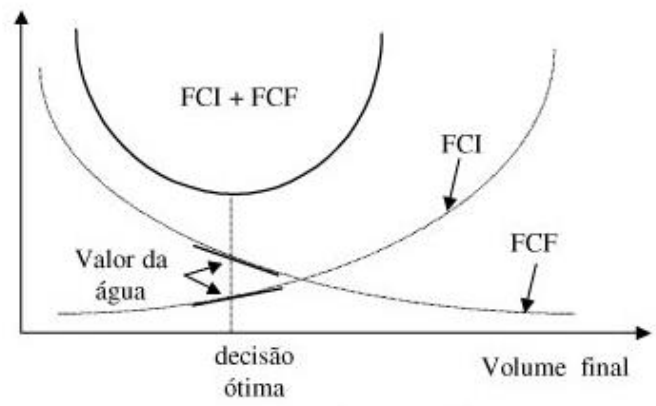

Figura 3- FCF do NEWAVE; onde FCI é a função de custo incial.

Fonte: [12].

Nesse modelo, a carga e a função de custo de déficit podem ser representadas em patamares e permite-se a consideração de limites de interligação entre os subsistemas [6].

O deck do modelo NEWAVE é disponibilizado mensalmente logo após o Programa Mensal de Operação (PMO). O conjunto de arquivos engloba todos os parâmetros que são utilizados na determinação da FCF, como por exemplo, dados técnicos de usinas térmicas e hidráulicas, informações sobre intercâmbios de energia, restrições elétricas e hidráulicas.

\subsection{DECOMP}

É o modelo de otimização utilizado para o horizonte de até 12 meses, com discretização semanal para o primeiro mês. $O$ modelo representa vazões previstas e a aleatoriedade das vazões após o primeiro mês através de uma árvore estocástica (cenários de vazões). Ao contrário do NEWAVE, no DECOMP, o parque gerador é representado individualmente (Usinas Hidrelétricas e Térmicas por subsistema).
O modelo DECOMP considera basicamente as chuvas que irão ocorrer no futuro para decisão do uso ou não da água dos reservatórios. Isto introduz uma relação entre a decisão de um modo de operação e suas consequências futuras conforme exemplificado na figura 2 [13].

O objetivo do modelo DECOMP é definir o despacho hidrotérmico que minimize o CMO ao longo do período de planejamento, este custo é composto pelo preço do combustível das usinas térmicas, intercâmbios de energia de outros subsistemas e penalidades pelo não atendimento à demanda. Algumas outras variáveis que compõem o preço são: a carga, as vazões e previsões de vazões, disponibilidades das usinas, limites de transmissão entre subsistemas e a FCF provinda do NEWAVE [13].

Semanalmente o ONS divulga o deck do DECOMP para semana vigente. O deck é composto por diversos arquivos que contém informações mais detalhadas, desde o histórico de vazões a informações mais específicas sobre carga por patamar e armazenamento individual de cada reservatório.

\section{VARIÁVEIS A SEREM \\ MONITORADAS PARA FORMAÇÃO DO PLD NO CURTO PRAZO}

Como dito na seção 2, o cálculo do PLD é determinado de maneira ex-ante, ou seja, é baseado em informações previstas. Durante a reunião do (PMO), realizado na sede do ONS na última quinta e sexta-feira do mês, são divulgadas as atividades de programação da operação, informações atualizadas sobre cronograma de expansão da geração e
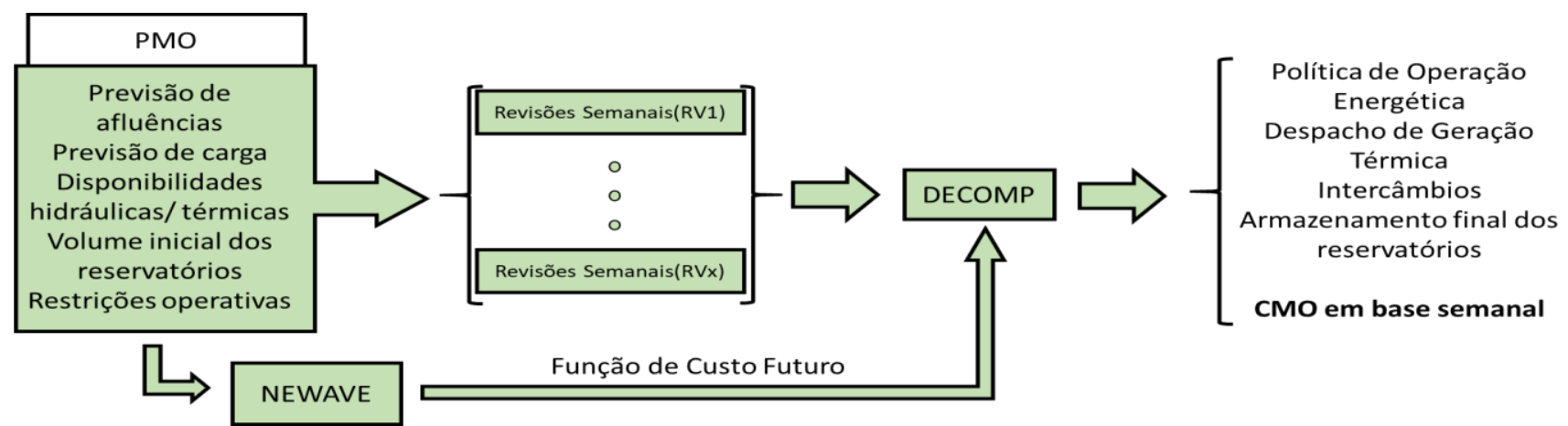

Figura 4: Fluxograma da Projeção do PLD.

Fonte: (MOURA FILHO, 2018). 
transmissão, estado atual do armazenamento dos reservatórios, previsões atualizadas de carga, análise das condições meteorológicas verificadas e previsões de afluências. Semanalmente esses dados podem ou não serem revisados pelo ONS (Revisões Semanais), ver fluxograma representado na figura 4 [14].

Baseado nisso, o agente comercializador de energia deve projetar o PLD para o curto prazo, uma vez que tenha que cumprir com contratos de compra/venda realizados anteriormente, para posterior liquidação na CCEE.

Para determinação do PLD semanal, algumas variáveis devem ser monitoradas constantemente, sendo as principais:

a) Afluências: A previsão de afluências para o mercado de curto prazo tem grande peso na formação do preço, uma vez que a elevação da vazão em usinas hidrelétricas faria que o DECOMP despachasse menos usinas térmicas, reduzindo o CMO. Dentro de uma comercializadora tal previsão é realizada diariamente através de modelos meteorológicos, como por exemplo ETA40km, BAM e GFS. Uma das entradas para o modelo DECOMP é a Energia Natural Afluente (ENA) por usina.

b) Carga: A função do modelo DECOMP é atender a demanda de energia do SIN, para isso, as comercializadoras estudam qual o comportamento dessa demanda para determinado período, considerando fatores elétricos, econômicos, climáticos, etc. Caso a carga aumente, devido a algum evento não programado, o despacho térmico pode ser necessário, elevando o custo marginal de operação. A carga é dividida em patamares de carga: leve, médio e pesado.

c) Armazenamento nos reservatórios: Outro fator importante para determinação do despacho é o nível dos reservatórios das usinas hidrelétricas. A projeção dos níveis depende de fatores como estratégia de operação para cada usina pelo ONS e previsão de afluências. Diariamente a ONS divulga 0 Relatório Diário HidráulicoHidrológica (RDH) contendendo a revisão das informações à cerca dos reservatórios de cada usina hidrelétrica;

Vale citar nesta seção o evento de afluências muito acima da média ocorrido na última semana do mês de maio e ao longo do mês de junho de
2017, em que o PLD sofreu uma queda abrupta devido à ocorrência de chuvas não previstas.

No PMO de maio, o ONS projetou 0 fechamento das afluências para o sistema SUL em cerca de $82 \%$ da Média de Longo Termo (MLT). Porém, devido à uma intensa frente fria que atingiu o sistema, a média mensal saltou para $168 \%$, como pode ser visto na figura 5 .

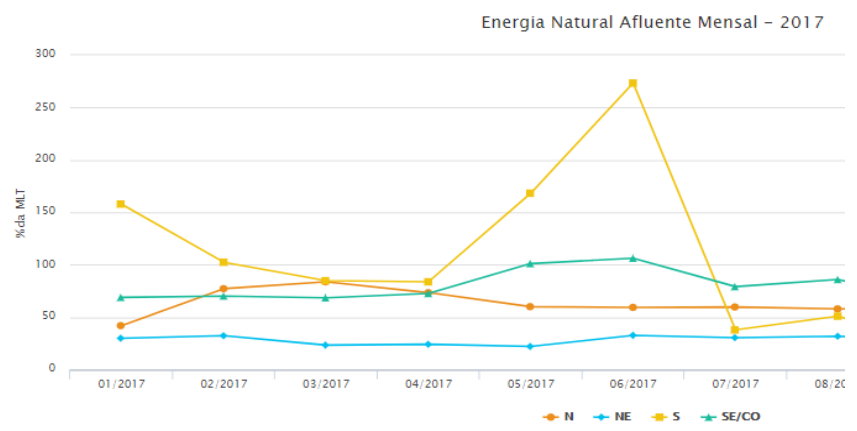

Figura 5: ENA de janeiro - julho 2017.

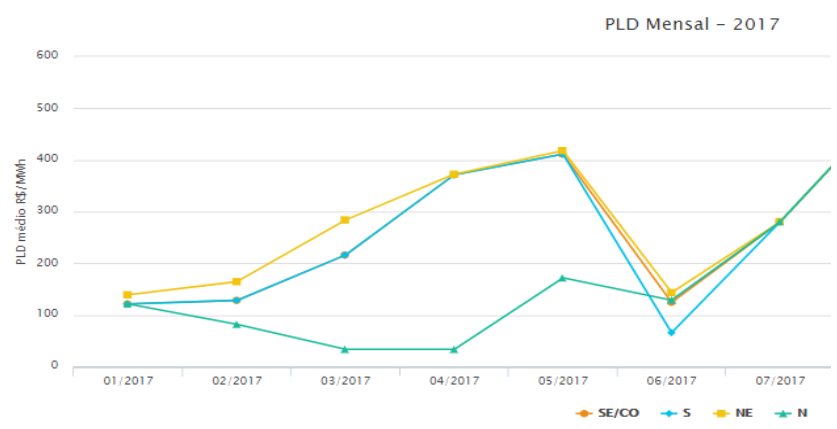

Figura 6: PLD de janeiro - julho 2017.

Essa afluência inesperada fez com que o PLD tivesse uma queda de $400 \%$ de um mês para outro impactando diretamente no preço médio mensal de maio, como podemos ver na figura 6 . Estas afluências impactaram também no mês de junho, reduzindo o preço para $R \$ 143,00$.

Para entender o impacto financeiro que essa queda no preço poderia ter causado a um agente comercializador, vamos supor que 0 comercializador comprou em maio $1 \mathrm{MWmed}$ do produto Junho por um valor de $\mathrm{R} \$ 350,00$ acreditando que o PLD iria subir ainda mais. Porém devido à chuva mencionada anteriormente o preço caiu para $R \$ 143,00$. Caso o agente esperasse até o final da liquidação do mês de junho para vender o produto teria um prejuízo de $\mathrm{R} \$ 149.000,00$ (Tabela 1). 
Tabela 1: Exemplo de Impacto financeiro

\begin{tabular}{|c|c|c|}
\hline & Comprou & Vendeu \\
\hline Preço & 350,00 & 143,00 \\
\hline Volume & 1,0 & 1,0 \\
\hline Horas & 720,0 & 720,0 \\
\hline Total & $R \$ 252.000,00$ & $R \$ 102.960,00$ \\
\hline Delta & $-R \$$ & $149.040,00$ \\
\hline
\end{tabular}

\section{RESULTADOS}

Nesta seção será apresentado um exemplo de simulação de projeção do PLD para as revisões semanais do mês de abril de 2018, alterando as variáveis de entrada mencionadas na seção 3 e utilizando os decks do DECOMP disponibilizados pela CCEE.

Inicialmente faz-se necessário entender como é a organização das semanas operativas divididas em revisões $(R V x)$ como pode ser visto na figura 7. Nessas revisões, ocorridas nas sextas-feiras, o ONS revisa e divulga os dados consolidados da semana operativa vigente e a previsão da semana seguinte, ajustando as informações divulgadas inicialmente no PMO.

Para projeção do PLD, foi utilizado o deck do DECOMP da revisão atual e o modificado para obter o PLD da semana seguinte, por exemplo, para projetar o preço da RV1 de abril utiliza-se o deck da RV0 e o modifica-se para que se tenha o preço da revisão 1 , analogamente para as outras revisões do mês de abril.

\begin{tabular}{|c|c|c|c|c|c|c|c|c|}
\hline Mês & Sábado & Domingo & Segunda & Terça & Quarta & Quinta & Sexta & Revisão \\
\hline$M$ arço/Abril & 24 & 25 & 26 & 27 & 28 & 29(PM O) & 30 (PM O) & RV4 M arço \\
\hline \multirow{4}{*}{ Abril } & 31 & 1 & 2 & 3 & 4 & 5 & 6 & RVO Abril \\
\hline & 7 & 8 & 9 & 10 & 11 & 12 & 13 & RV1 Abril \\
\hline & 14 & 15 & 16 & 17 & 18 & 19 & 20 & RV2 Abril \\
\hline & 21 & 22 & 23 & 24 & 25 & 26 (PMO) & 27(PMO) & RV3 A Abril \\
\hline Abril/M aio & 28 & 29 & 30 & 1 & 2 & 3 & 4 & RVO Maio \\
\hline
\end{tabular}

Figura 7: Organização das Semanas Operativas. Fonte: (MOURA FILHO, 2018).

No PMO de abril, ocorrido nos dias 29 e 30 de março, o ONS divulgou os decks do NEWAVE e DECOMP juntamente com a programação da operação, calendário de expansão da geração e transmissão, armazenamento dos reservatórios, previsões atualizadas de carga, afluências verificadas e previsões.
Como mostrado na Tabela 2, na RV0 de abril divulgada no dia 30 de março, o ONS considerou um fechamento mensal de ENA para o subsistema Sul de $152 \%$ da MLT influenciando diretamente no PLD dos sistemas Sudeste e Sul, que foi o piso regulatório, $\mathrm{R} \$ 40,16$.

Para projeção do preço por parte do agente comercializador é necessário realizar estudos e monitoramento das afluências, no cenário de sensibilidade as chuvas não seriam tão intensas como o ONS considerou, sendo simulada com uma MLT de 106\%, simulou-se também um eventual aumento de carga no sistema Sudeste, elevando o PLD em 65\%.

Tabela 2: Sensibilidade para RV0.

\begin{tabular}{|c|c|c|c|c|c|}
\hline \multirow{6}{*}{$\begin{array}{c}\text { ONS } \\
\text { ENA Sul } \\
(152 \% M L T)\end{array}$} & \multirow{6}{*}{$\begin{array}{c}\text { SENSIBILIDADE } \\
\text { ENA Sul } \\
\text { (106\%MLT) } \\
\text { Carga Sudeste } \\
\text { (+100M Wmed) }\end{array}$} & \multirow{2}{*}{ Subsistema } & \multicolumn{3}{|c|}{ PLD RV0 (R\$/MWh) } \\
\hline & & & ONS & Sensibilidade & Delta \\
\hline & & Sudeste & 40,16 & 66,33 & $65,2 \%$ \\
\hline & & Sul & 40,16 & 66,33 & $65,2 \%$ \\
\hline & & Nordeste & 40,16 & 66,33 & $65,2 \%$ \\
\hline & & Norte & 40,16 & 66,33 & $65,2 \%$ \\
\hline
\end{tabular}

Como mostrado na tabela 3, para a Revisão 1, o ONS revisou as informações divulgadas no PMO e considerou o fechamento mensal do sistema Sudeste em $93 \%$ da MLT e um armazenamento inicial para semana da revisão de $44 \%$. Para o caso de sensibilidade, simulou-se uma ENA mais baixa, cerca de $90 \%$, o nível mais baixo para o reservatório, e uma eventual elevação na carga do sistema. Esses fatores elevaram 0 PLD em quase $22 \%$ nos subsistemas SE/CO e $8,4 \%$ no subsistema Norte.

Tabela 3: Sensibilidade para RV1.

\begin{tabular}{|c|c|c|c|c|c|}
\hline \multirow{6}{*}{$\begin{array}{c}\text { ONS } \\
\text { ENA Sudeste } \\
(93 \% \mathrm{MLT}) \\
\text { EAR Sudeste } \\
(44,2 \%)\end{array}$} & \multirow{6}{*}{$\begin{array}{c}\text { SENSIBILIDADE } \\
\text { ENA Sudeste } \\
(90 \% M L T) \\
\text { EAR Sudeste } \\
(42 \%) \\
\text { Caga Sudeste } \\
\text { (+200M Wmed) }\end{array}$} & \multirow{2}{*}{ Subsistema } & \multicolumn{3}{|c|}{ PLD RV1(R\$/MWh) } \\
\hline & & & ONS & Sensibilidade & Delta \\
\hline & & Sudeste & 84,83 & 103,45 & $21,9 \%$ \\
\hline & & Sul & 84,83 & 103,45 & $21,9 \%$ \\
\hline & & Nordeste & 106,83 & 106,83 & $0,0 \%$ \\
\hline & & Norte & 78,03 & 84,6 & $8,4 \%$ \\
\hline
\end{tabular}

Para Revisão 2, na Tabela 4, foi considerado a projeção do fechamento para as afluências do mês de abril no sistema Sudeste em 93\% da MLT e um armazenamento de 46\%. Como sensibilidade para projeção do preço, consideramos uma afluência que elevasse a ENA para $97 \%$ da MLT, consideramos também que essa chuva elevou o armazenamento em $2 \%$. Caso esse cenário de chuvas se configurasse, 0 preço teria uma redução de $15,0 \%$ no SE/CO e $48,5 \%$ no Norte, este último voltando para o piso regulatório. 
Tabela 4: Sensibilidade para RV2.

\begin{tabular}{|c|c|c|c|c|c|}
\hline \multirow{6}{*}{$\begin{array}{c}\text { ONS } \\
\text { ENA Sudeste } \\
(93 \% \mathrm{MLT}) \\
\text { EAR Sudeste } \\
(44 \%)\end{array}$} & \multirow{6}{*}{$\begin{array}{c}\text { SENSIBILIDADE } \\
\text { ENA Sudeste } \\
\text { (97\%MLT) } \\
\text { EAR Sudeste } \\
(46 \%)\end{array}$} & \multirow{2}{*}{ Subsistema } & \multicolumn{3}{|c|}{ PLD RV2 (R\$/MWh) } \\
\hline & & & ONS & Sensibilidade & Delta \\
\hline & & Sudeste & 122,73 & 104,28 & $-15,0 \%$ \\
\hline & & Sul & 122,73 & 104,28 & $-15,0 \%$ \\
\hline & & Nordeste & 122,73 & 106,83 & $-13,0 \%$ \\
\hline & & Norte & 78,03 & 40,16 & $-48,5 \%$ \\
\hline
\end{tabular}

Na Tabela 5, temos um caso de sensibilidade para Revisão 3 do mês de abril. Nesse caso, o ONS ajustou sua previsão de fechamento para o sistema Sul em $100 \%$ e simulamos com uma elevação de afluência para $120 \%$ da MLT, somado a isso, uma diminuição de carga em 200MWmed no sistema Sudeste. Com estes novos inputs, tivemos uma redução de $23,5 \%$ do preço no SE/CO e Nordeste, sem alteração no sistema Norte.

Tabela 5: Sensibilidade para RV3.

\begin{tabular}{|c|c|c|c|c|c|}
\hline \multirow{6}{*}{$\begin{array}{c}\text { ONS } \\
\text { ENA Sul } \\
(100 \% \mathrm{MLT})\end{array}$} & \multirow{6}{*}{$\begin{array}{c}\text { SENSIBILIDADE } \\
\text { ENA Sul } \\
\text { (120\%MLT) } \\
\text { Carga Sudeste } \\
\text { (-200M Wmed) }\end{array}$} & \multirow{2}{*}{ Subsistema } & \multicolumn{3}{|c|}{ PLD RV3 (R\$/MWh) } \\
\hline & & & ONS & Sensibilidade & Delta \\
\hline & & Sudeste & 135,38 & 103,57 & $-23,5 \%$ \\
\hline & & Sul & 135,38 & 103,57 & $-23,5 \%$ \\
\hline & & Nordeste & 135,38 & 103,57 & $-23,5 \%$ \\
\hline & & Norte & 40,16 & 40,16 & $0,0 \%$ \\
\hline
\end{tabular}

Outra ferramenta utilizada pelo agente comercializador na projeção do preço é a elaboração de uma matriz de preços. Para essa matriz, na maioria das vezes são simulados intervalos de ENA em \% da MLT SE/CO x Sul, pois são os sistemas que mais influenciam na formação do preço. Simulamos um caso para Revisão 3, como visto na Tabela 6.

Tabela 6: Matriz de Sensibilidade SE/CO x SUL

\begin{tabular}{|c|c|c|c|c|}
\hline \multirow{2}{*}{ RV3 } & $\mathrm{R} \$ / \mathrm{M} \mathrm{Wh}$ & \multicolumn{3}{|c|}{ Sul(\%MLT) 1} \\
\hline & ENA & $95 \%$ & $100 \%$ & $105 \%$ \\
\hline \multirow{3}{*}{ Sudeste(\%MLT) } & $90 \%$ & 143,91 & 137,00 & 120,56 \\
\hline & $92 \%$ & 132,69 & 128.50 & 110,25 \\
\hline & $95 \%$ & 118,07 & 110,00 & 104,26 \\
\hline
\end{tabular}

Como visto na Tabela 5 da Revisão 3, o fechamento mensal de ENA no Sul foi realizado em $100 \%$ e $91,5 \%$ para subsistema o SE/CO. Podemos ver que na matriz de sensibilidade elaborada ainda durante a semana da Revisão 2, o valor do PLD do SE/CO da Revisão 3 de R\$ 133,38 já estava mapeado na matriz.

\section{CONCLUSÃO}

Baseado nos eventos históricos e nas simulações realizadas no tópico anterior, concluise, portanto, que o monitoramento das variáveis que compõem o PLD é crucial para a projeção dos preços do mercado de curto prazo de energia elétrica no Brasil. O acompanhamento das afluências, do nível dos reservatórios e da carga deve ser constante. Outras variáveis que possuem pouca volatilidade, mas também devem ser monitoradas são, a expansão da geração térmica e hídrica; bem como as capacidades de intercâmbio de energia entre os subsistemas.

O modelo DESSEM que atualmente está em processo de validação, terá discretização horária e poderá representar de maneira mais detalhada tanto aspectos relacionados à rede elétrica como aspectos relacionados à operação das usinas hidroelétricas, termoelétricas e demais componentes do sistema, aumentando assim a granularidade das análises de sensibilidade para a projeção futura do PLD.

\section{REFERÊNCIAS}

[1] PALOMINO, J. M. G. Formação de Preço de energia elétrica gerada por biomassa no ambiente de Contratação Livre Brasileiro: Uma abordagem computacional baseada em agentes. 2009. 118 f. Dissertação (Mestrado em Economia, Administração e Contabilidade) Faculdade de Economia, administração e Contabilidade, Universidade de São Paulo, Ribeirão Preto, 2009.

[2] MAGALHÃES, G. D. S. C. Comercialização de Energia Elétrica no Ambiente de Contratação Livre: Uma análise Regulatório-Institucional a partir dos contratos de compra e venda de energia elétrica. 2009. 140 f. Dissertação (Mestrado.em Engenharia) - Universidade de São Paulo, São Paulo, 2009.

[3] TATEMOTO, K. A. Energia incentivada: Uma análise integrada dos aspectos regulatórios, de comercialização e de sustentabilidade. 2013. 162 f. Dissertação (Mestrado em Engenharia e Energia e Automoção Elétricas) - Universidade de São Paulo, São Paulo, 2013.

[4] JANUÁRIO, A. C. V. O Mercado de Energia Elétrica de fontes incentivadas: Proposta para sua expansão e implicações na câmara de comercialização de energia elétrica. 2007. $121 \mathrm{f}$. Dissertação (Mestrado de Engenharia e Energia e Automoção 
Elétricas) . Universidade de São Paulo, São Paulo, 2007.

[5] TOLMASQUIM, M. T. Novo Modelo do Setor Elétrico Brasileiro. 2. ed. Rio de Janeiro: Synergia, 2015.

[6] CCEE. Preços. Câmara de Comercialização de Energia, 2017. Disponível em: https://www.ccee.org.br/portal/. Acesso em: 19 Maio 2018.

[7] CEPEL. Manual de Referência - Relatório Técnico - Modelo Decomp. Rio de Janeiro: CEPEL, 2001.

[8] RAMOS, T. P. Modelo Individualizado de Usinas Hidréletricas Baseado em Técnias de Programação não Linear Integrado com o Modelo de Decisão Estratégica. 2011. 95 f. Dissertação (Mestrado Engenharia Elétrica) - Universidade Federal de Juiz de Fora, Juiz de Fora, 2011.

[9] TOSCANO, A. E. Comparação entre os modelos NEWAVE e ODIN no planejamento energético do Sistema Interligado Nacional. 2009. $111 \mathrm{f}$. Dissertação (Mestrado em Engenharia Elétrica) - Universidade Estadual de Campinas, Campinas, 2009.

[10] CEPEL. Manual de Referência - Relatório Técnico - Modelo Newave.Centro de Pesquisas de Energia Elétrica. Rio de Janeiro: CEPEL. 2001.

[11] MARCATO, R. M. Redistribuição ótima em patamares de carga da geração mensal de usinas hidrelétricas. 2007.

Dissertação (Mestrado em Métodos Matemáticos em Energia) - Instituto Nacional de Matemática Aplicada. Rio de Janeiro. 2007.

[12] SIMÕES, M. D. D. P.; GOMES, L. L. Decisão de sazonalização de contratos de fornecimento de energia elétrica no Brasil através da otimização da medida Ômega $(\Omega)$. Revista Eletrônica de Administração, Porto Alegre, v. 17, n. 1, abr. 2011.

[13] CEPEL. Manual de Referência - Relatório Técnico - Modelo Decomp. CEPEL. Rio de Janeiro: CEPEL, 2001.

[14] ONS. Programa Mensal de Operação PMO. Operador Nacional do Sistema Elétrico, 2018. Disponivel em: <http://ons.org.br/pt/paginas/energia-nofuturo/programacao-da-operacao $>$. Acesso em: 03 Junho 2018. 